\title{
Effects of Food Habits and Lifestyle on Prevalence of Overweight/Obesity among Schoolchildren in Taif Area, KSA
}

\author{
Fayez Hamam ${ }^{1}$, Ahmed Eldalo', Mohammad Khaleel ${ }^{2}$, Abdullah Alwagdani ${ }^{3}$, Ahmed Alqarni ${ }^{3}$, \\ Bandr Daghas ${ }^{3}$, Msfer Alharthi ${ }^{3}$, Huda Alharthy ${ }^{3}$, Samah Hassen ${ }^{3}$, Abrar Alsofiany3, \\ Sahar Alotaibie ${ }^{3}$
}

\footnotetext{
${ }^{1}$ Department of Pharmacology and Toxicology, College of Pharmacy, Taif University, Taif, Kingdom of Saudi Arabia ${ }^{2}$ Department of Pharmaceutics and Pharmaceutical Technology, College of Pharmacy, Taif University, Taif, Kingdom of Saudi Arabia

${ }^{3}$ Department of Clinical Pharmacy, College of Pharmacy, Taif University, Taif, Kingdom of Saudi Arabia

Email: ^fhamam@yahoo.com, f.hamam@tu.edu.sa
}

How to cite this paper: Hamam, F., Eldalo, A., Khaleel, M., Alwagdani, A., Alqarni, A., Daghas, B., Alharthi, M., Alharthy, H., Hassen, S., Alsofiany, A. and Alotaibie, S. (2017) Effects of Food Habits and Lifestyle on Prevalence of Overweight/Obesity among Schoolchildren in Taif Area, KSA. Food and Nutrition Sciences, 8, 196-211.

https://doi.org/10.4236/fns.2017.82013

Received: January 4, 2017

Accepted: February 4, 2017

Published: February 7, 2017

Copyright $\odot 2017$ by authors and Scientific Research Publishing Inc. This work is licensed under the Creative Commons Attribution International License (CC BY 4.0).

http://creativecommons.org/licenses/by/4.0/

\begin{abstract}
Background/Objectives: The Saudi population has experienced significant transformations in their lifestyle which could be attributed to several factors. Aims: This work sought to determine effects of food habits and lifestyle on prevalence of overweight (OW)/obesity (OB) among schoolchildren in Taif City, KSA. Materials and Methods: A cross-sectional study was carried out using well-designed questionnaire. Pretested questionnaire was randomly distributed among schoolchildren in Taif city. Body mass index and waist circumference were used as tools for assessing nutritional status of schoolchildren. Results: A total of 2027 students filled the questionnaire with a mean age of $15.83 \pm 2.18$ years. The prevalence of $\mathrm{OW}$ and $\mathrm{OB}$ was $14.6 \%$ and $12.9 \%$, respectively, while the combined one was $27.5 \%$. The combined prevalence of OW and OB was significantly higher among boys than girls (31.9 vs. 21.9). Schoolchildren from high income families had high rate of OW and OB. Smoking, stress, TV viewing, daylight and night sleep had no effects on BMI, while only smoking of a family member and stress had considerable relationship with W_C. Unfortunately, $52.2 \%$ of students admitted that they were physically inactive but this effect was insignificant for both indicators. Students skipped breakfasts constitute $18.8 \%$ of subjects included but link with BMI and W_C was statistically insignificant. Regular soft or diet drinks had significant effects on BMI. Results showed considerable link between consumption of fruits/vegetables, grains and W_C. Conclusions: The prevalence of combined OW/OB among schoolchildren was $27.5 \%$. Family income, participant's gender, intake of regular soft or diet drinks had significant effects on
\end{abstract}


BMI. While, stress, smoking of a family member, and uptake of fruits and vegetables showed considerable relationship with $\mathrm{W}_{-} \mathrm{C}$.

\section{Keywords}

Overweight, Obesity, Body Mass Index, Waist Circumference, Food Habits, Lifestyle

\section{Introduction}

KSA is a country that a large percent of population is youth [1]. The population of KSA experienced significant transformations in their lifestyle [2]. The epidemic of OW and $\mathrm{OB}$ among children and adolescent is alarming in KSA, since there is a tendency of OW and obese children to remain OW and obese as adults [3]. The impact of OW and OB on children's health is manifested in terms of increase risk of heart disorders, hypertension, lessening of flow of cerebral blood, and asymptomatic coronary heart atherosclerosis. OB and OW also affect the endocrine (i.e. high risk of type 2 diabetes mellitus and hypogonadism). The socio-economic consequences of $\mathrm{OB}$ and $\mathrm{OW}$ include low self-esteem, poor body image, and limitation in mobility and production [4]. Several research works showed that food habits are the most crucial factors affecting the health status of schoolchildren in KSA. For example, Collison et al. [5] conducted a cohort study of 9433 schoolchildren aged 10 to 19 in the capital city (Riyadh). The researchers found that boys had higher rates of OB than girls. Abahussain [6] found that $28 \%$ of school girls in the Eastern Province of KSA were overweight and obese. Omer et al. [2] found that $11 \%$ of Al-Baha (South Western part of KSA) schoolchildren were OW or obese. Al-Reethaiaa et al. [7] found that the prevalence of $\mathrm{OW}$ and $\mathrm{OB}$ was $21.8 \%$ and $15.7 \%$, respectively among male Health Sciences students at Qassim University, KSA [7]. Amin et al. [8] indicated that the prevalence of OW and $\mathrm{OB}$ among schoolchildren in Al-Hassa (Eastern Province, KSA) was $14.2 \%$ and 9.7\%, respectively. El-Quadh [9] showed that the prevalence of $\mathrm{OB}$ among male Health Sciences students was higher than females (16.7\% VS. 6.7\%) in the North Western City of Tabuk (KSA). El-Hazmi and Warsy [10] demonstrated that the combined prevalence of OW and OB in Saudi boys and girls is $16.7 \%$ and $19.4 \%$, respectively. Farghaly [11] found that the most consumed diet by schoolchildren in Abha City (KSA) contained higher quantity of carbohydrates and lower amount of fiber. Al-Oboudi [12] showed that $26.0 \%$ of school girls in Riyadh were either OW and/or OB. Almuhnna et al. [13] found considerable $(P<0.05)$ link between OB and uptake of fast foods. Abahussain [14] did not find a significant change in BMI among Saudi school girls between 1997 and 2007. Al-Shehri [15] confirmed positive relationship between intake of unhealthy foods and body mass. In conclusion, many studies were conducted in KSA to assess nutritional status among residents of the kingdom. However, none of the previously mentioned studies, at least to the best of 
our knowledge, tried to assess nutritional status among schoolchildren in Taif area. The objectives were 1) to investigate effects of students' demographic characteristics on BMI and W_C among schoolchildren in Taif City (Western part of KSA);2) to assess the association between students' eating habits and BMI and W_C, and 3) to evaluate the link between lifestyle of participants and both indicators.

This study determined the prevalence of overweight/obesity and relationship with eating habits and lifestyles among schoolchildren in Taif area. Shedding light on the nature of the link between overweight/obesity and food habits as well as lifestyle will be of great significance in the prevention and treatment of several diseases related malnutrition, such as obesity, heart diseases, diabetes mellitus, and hypertension, among others. Furthermore, this study will be the basis of the second stage which will try to initiate a partnership among different parties, including schools, College of Pharmacy at Taif University, parents, department of school health services, and health-care giver. Furthermore, this study will be the basis for initiating and implementing a health education program that will encourage students to take steps toward preventive actions, including limiting less-healthy diets and endorsing healthy foods in their meals.

\section{Materials and Methods}

\subsection{Study Design and Study Population}

A cross-sectional study design was carried out in the period from Feb. to Oct. 2015. This study was conducted among schoolchildren attending preparatory and secondary schools in Taif City, KSA. A suitable sampling technique was adopted. Schools were carefully selected from different areas of Taif. A total of 5000 questionnaire was distributed to representative schools in the four different regions of Taif city. A consent form was explained by members of the research team and students were asked to bring signed consent form their parents before participating in this study. It should be noted that filling out questionnaires was done under direct supervision of the research team members. Thus, the pretested questionnaire was explained and basic information about food guide and tips to get active was presented before a small group of students (e.g. grade 7 class composed of 20 - 25 students).

\subsection{Study Instrument}

A structured well-prepared questionnaire was designed. The questionnaire composed of two sections. The first section was designed to obtain students' demographic characteristics such as: gender, area of residence, family income etc. This section also collected anthropometric measurements include: height (meters), weight $(\mathrm{kg})$, and waist circumference $(\mathrm{cm})$. Anthropometric measurements were measured by members of the research team to ensure accuracy of measurements. Height of participants was determined to the nearest $0.2 \mathrm{~cm}$ using a measuring scale equipped with sliding head part attached to a measuring rod. Body mass was determined to the closest $0.1 \mathrm{~kg}$. After that BMI was calculated using stan- 
dard equation. The calculated BMIs were classified to underweight, normal weight, OW and obese depending on the US Centers for Disease Control and Prevention (CDC) growth charts for classification of OW and OB among children. According to this chart, children were classified as OW when their percentile located between $\geq 85^{\text {th }}$ to $<95^{\text {th }}$, and as obese when their percentile located $\geq 95^{\text {th }}$. Those located between $\geq 5^{\text {th }}$ to $<85^{\text {th }}$ percentile were classified normal. When the percentile $<5^{\text {th }}$ children were considered normal [16]. Even though BMI is a widely used screening methods, it has major restrictions since it isn't able to differentiate between lean and fat (muscle) mass; it has been proposed that percentage of body fat plays a more significant role in distinguishing healthy from unhealthy subjects [17]. Thus, waist circumference has been used as another tool for assessing nutritional status. The cut-off points for classification of $\mathrm{OW}$ and $\mathrm{OB}$ based on W_C were determined according to the International Diabetes Federation (IDF) [18]. The second section collected data about participants' lifestyle and food habits.

\subsection{Ethical Consideration}

This study had obtained ethical approval (No: 83056) from the Ethical Committee at Taif University, KSA. Informed verbal consent was obtained from every student before filling the study tool.

\subsection{Sampling Technique and Sample Size}

A total of 2027 questionnaires were randomly distributed to students in representative schools. It should be noted that filling out questionnaires was done under direct supervision of the researchers.

\subsection{Analysis of Data}

Data was analyzed using the statistical package for social sciences (IBM SPSS, version 22, Armonk, NY: IBM Corp.). Means and frequencies as percentages were used to describe different variables. Chi-square analysis was employed to examine the association between the participants' demographic attributes, lifestyle, eating habits and BMI, and W_C. The significance of the differences was determined at $P$ value $<0.05$.

\section{Results}

\subsection{Demographic Characters}

A total of 2027 students from both genders filled the questionnaire with a mean age of $15.83 \pm 2.18$ years. The response rate was $100 \%$. Out of them 293 (14.6\%) were OW and $260(12.9 \%)$ were obese. The prevalence of OW + OB was 553 (27.5\%), while $1808(89.3 \%)$ were at no risk when W_C was used as an assessment tool. More than half 1137 (56.1\%) of the subjects included were male and the majority $1565(77.2 \%)$ were Saudi students. There were significant associations $(P<0.001)$ between participants' gender and their BMI, $31.9 \%$ of males 
and only $21.9 \%$ of females were OW and obese. The OB percentage among students comes from low-income families were significantly lower (13\%) from those come from high-income families $(15.2 \%)(P<0.001)$. Children live in the Sothern part of Taif City had the highest prevalence of OW (17.4\%) and OB (18.4\%), furthermore, the combined rate of OW and OB $(35.8 \%)$ was also the highest among schoolchildren live in the Southern part compared to those live in other regions of Taif City (Table 1 ).

\subsection{Life Style}

Table 2 shows the effects of lifestyle and environmental exposure on BMI and W_C. Although the majority of participants were non-smokers 1847 (91.8\%), their family members smoking rate was high 912 (45\%). Smoking neither affected

Table 1. Demographic characteristics and their effects on BMI and W_C among schoolchildren living in Taif City, KSA.

\begin{tabular}{|c|c|c|c|c|c|c|c|c|c|c|}
\hline \multirow{2}{*}{\multicolumn{2}{|c|}{ Characters }} & \multirow[b]{2}{*}{ Freq. (\%) } & \multicolumn{5}{|c|}{ Body mass classification } & \multicolumn{3}{|c|}{ Waist Circumference } \\
\hline & & & Under-weight & $\begin{array}{l}\text { Normal } \\
\text { weight }\end{array}$ & Over-weight & $\mathrm{OB}$ & $P$-value & No risk & Risk & $\mathrm{p}$-value \\
\hline \multirow[b]{2}{*}{ Gender } & Male & $1137(56.1 \%)$ & $228(20.1 \%)$ & $545(48.1 \%)$ & $163(14.4 \%)$ & $198(17.5 \%)$ & \multirow[b]{2}{*}{$<0.001$} & $956(84.2 \%)$ & $179(15.8 \%)$ & \multirow{2}{*}{$<0.001$} \\
\hline & Female & $890(43.9 \%)$ & $75(8.5 \%)$ & $612(69.6 \%)$ & $130(14.8 \%)$ & $62(7.1 \%)$ & & $852(95.7 \%)$ & $38(4.3 \%)$ & \\
\hline \multirow{2}{*}{ Nationality } & Saudi & $1565(77.2 \%)$ & $235(15.1 \%)$ & $884(56.9 \%)$ & $232(14.9 \%)$ & $202(13.0 \%)$ & \multirow{2}{*}{0.927} & $1405(89.8 \%)$ & $159(10.2 \%)$ & \multirow{2}{*}{0.317} \\
\hline & Non-Saudi & $460(22.7 \%)$ & $68(14.8 \%)$ & $271(59.2 \%)$ & $61(13.3 \%)$ & $58(12.7 \%)$ & & $401(87.4 \%)$ & $58(12.6 \%)$ & \\
\hline \multirow{6}{*}{ Grade } & 7 & $354(17.5 \%)$ & $56(16 \%)$ & $192(54.7 \%)$ & $53(15.1 \%)$ & $50(14.2 \%)$ & \multirow{6}{*}{0.119} & $326(92.4 \%)$ & $27(7.6 \%)$ & \multirow{6}{*}{0.092} \\
\hline & 8 & $355(17.5 \%)$ & $55(15.5 \%)$ & $196(55.4 \%)$ & $52(14.7 \%)$ & $51(14.4 \%)$ & & $306(86.2 \%)$ & $49(13.8 \%)$ & \\
\hline & 9 & $247(12.2 \%)$ & $48(19.5 \%)$ & $129(52.4 \%)$ & $37(15 \%)$ & $32(13 \%)$ & & $214(86.6 \%)$ & $33(13.4 \%)$ & \\
\hline & 10 & $321(15.8 \%)$ & $39(12.1 \%)$ & $180(56.1 \%)$ & $50(15.6 \%)$ & $52(16.2 \%)$ & & $287(89.4 \%)$ & $34(10.6 \%)$ & \\
\hline & 11 & $438(21.6 \%)$ & $56(12.9 \%)$ & $270(62.4 \%)$ & $58(13.4 \%)$ & $49(11.3 \%)$ & & $399(91.1 \%)$ & $39(8.9 \%)$ & \\
\hline & 12 & $307(15.1 \%)$ & $49(16.2 \%)$ & 187 (61.7\%) & $41(13.5 \%)$ & $26(8.6 \%)$ & & $272(88.6 \%)$ & $35(11.4 \%)$ & \\
\hline \multirow{3}{*}{$\begin{array}{l}\text { Family } \\
\text { income }\end{array}$} & $<$ SAR 5000 & $450(22.2 \%)$ & $65(\%)$ & $266(59.8 \%)$ & $56(12.6 \%)$ & $58(13 \%)$ & \multirow{3}{*}{0.006} & $399(88 \%)$ & $50(11.1 \%)$ & \multirow{3}{*}{0.556} \\
\hline & $\begin{array}{c}\text { SAR } \\
5000-10,000\end{array}$ & $622(30.7 \%)$ & $110(17.7 \%)$ & $344(55.5 \%)$ & $98(15.8 \%)$ & $68(11 \%)$ & & $547(88.1 \%)$ & 74 (11.9\%) & \\
\hline & $\begin{array}{c}\text { SAR } \\
10,000-15,000\end{array}$ & $451(22.2 \%)$ & $70(15.6 \%)$ & $264(58.9 \%)$ & $57(12.7 \%)$ & $57(12.7 \%)$ & & $408(90.5 \%)$ & $43(9.5 \%)$ & \\
\hline \multirow{5}{*}{$\begin{array}{l}\text { Location of } \\
\text { residency }\end{array}$} & Taif North & $400(19.7 \%)$ & $66(16.5 \%)$ & $241(60.4 \%)$ & $48(12 \%)$ & $44(11 \%)$ & & $367(91.8 \%)$ & $33(8.3 \%)$ & \multirow{5}{*}{0.248} \\
\hline & Taif South & $323(15.9 \%)$ & $26(8.1 \%)$ & $180(56.1 \%)$ & $56(17.4 \%)$ & $59(18.4 \%)$ & & $285(88.2 \%)$ & $38(11.8 \%)$ & \\
\hline & Taif East & $549(27.1 \%)$ & $71(\% 13.1)$ & $343(63.4 \%)$ & $77(14.2 \%)$ & $50(9.2 \%)$ & $<0.001$ & $494(90.1 \%)$ & $54(9.9 \%)$ & \\
\hline & Taif West & $750(37.0 \%)$ & $139(18.6 \%)$ & $390(52.2 \%)$ & $111(14 \%)$ & $107(14.3 \%)$ & & $658(87.9 \%)$ & $91(12.1 \%)$ & \\
\hline & Total & & $303(15.1 \%)$ & 1157 (57.5\%) & $293(14.6 \%)$ & $260(12.9 \%)$ & & $1808(89.3 \%)$ & $217(10.7 \%)$ & \\
\hline
\end{tabular}

Freq. $(\%)=$ Frequency (percentage); SAR $=$ Saudi Arabia Riyals. 
Table 2. Effects of lifestyle and environmental exposure on BMI and W_C among schoolchildren living in Taif City, KSA.

\begin{tabular}{|c|c|c|c|c|c|c|c|c|c|c|}
\hline & & \multirow[b]{2}{*}{ Freq. (\%) } & \multicolumn{5}{|c|}{ Body mass classification } & \multicolumn{3}{|c|}{ Waist Circumference } \\
\hline & & & Under-weight & $\begin{array}{c}\text { Normal } \\
\text { weight }\end{array}$ & Over-weight & OB & $P$-value & No risk & Risk & p-value \\
\hline \multirow{3}{*}{ Smoking } & Yes & $164(8.1 \%)$ & $281(17.1 \%)$ & $77(47 \%)$ & $33(20.1 \%)$ & $26(15.9 \%)$ & \multirow{3}{*}{0.100} & $142(86.6 \%)$ & $22(12.4 \%)$ & \multirow{3}{*}{0.436} \\
\hline & & & & & & & & & & \\
\hline & No & $1847(91.8 \%)$ & $274(14.8 \%)$ & $1079(58.4 \%)$ & $260(14.1 \%)$ & $234(12.7 \%)$ & & $1664(89.5 \%)$ & $195(10.5 \%$ & \\
\hline \multirow{3}{*}{$\begin{array}{c}\text { Smoking } \\
\text { of a family } \\
\text { member }\end{array}$} & Yes & $912(45 \%)$ & $143(15.8 \%)$ & $517(57.1 \%)$ & $130(14.4 \%)$ & $115(12.7 \%)$ & \multirow{3}{*}{0.587} & $836(91.8 \%)$ & $75(8.2 \%)$ & \multirow{3}{*}{0.013} \\
\hline & & & & & & & & & & \\
\hline & No & $1110(55 \%)$ & $159(14.4 \%)$ & $638(57.8 \%)$ & $163(14.8 \%)$ & $143(13 \%)$ & & $967(87.2 \%)$ & $142(12.8 \%)$ & \\
\hline \multirow{2}{*}{$\begin{array}{c}\text { Role of } \\
\text { stress }\end{array}$} & Yes & $955(47.1 \%)$ & $135(14.3 \%)$ & $554(58.7 \%)$ & $141(14.9 \%)$ & $114(12.1 \%)$ & \multirow{2}{*}{0.541} & $868(90.9 \%)$ & $87(9.1 \%)$ & \multirow{2}{*}{0.030} \\
\hline & No & $1045(51.6 \%)$ & $164(15.7 \%)$ & $586(56.2 \%)$ & $151(14.5 \%)$ & $141(13.5 \%)$ & & $914(87.6 \%)$ & $129(12.4 \%)$ & \\
\hline \multirow{2}{*}{$\begin{array}{l}\text { Physical } \\
\text { activity }\end{array}$} & Yes & $961(47.4 \%)$ & $137(14.4 \%)$ & $569(59.6 \%)$ & $136(14.3 \%)$ & $112(11.7 \%)$ & \multirow{2}{*}{0.470} & $848(88.2 \%)$ & $113(11.8 \%)$ & \multirow{2}{*}{0.116} \\
\hline & No & $1058(52.2 \%)$ & $164(15.6 \%)$ & $585(55.7 \%)$ & $155(14.7 \%)$ & $147(14 \%)$ & & $954(90.3 \%)$ & $102(9.7 \%)$ & \\
\hline \multirow{6}{*}{$\begin{array}{c}\text { Duration } \\
\text { of Physical } \\
\text { activity }\end{array}$} & $<1 \mathrm{~h}$ & $251(12.4 \%)$ & $38(15.3 \%)$ & $140(56.2 \%)$ & $41(16.5 \%)$ & $30(12 \%)$ & \multirow{6}{*}{0.665} & $226(90 \%)$ & $25(10 \%)$ & \multirow{6}{*}{0.233} \\
\hline & $2 \mathrm{~h}$ or less & $192(9.5 \%)$ & $30(15.7 \%)$ & $124(64.9 \%)$ & $17(8.9 \%)$ & $20(10.5 \%)$ & & $172(89.6 \%)$ & $20(10.4 \%)$ & \\
\hline & & & & & & & & & & \\
\hline & $3 \mathrm{~h}$ a wk & $137(6.8 \%)$ & $20(14.8 \%)$ & $76(56.3 \%)$ & $23(17 \%)$ & $16(11.9 \%)$ & & $117(85.4 \%)$ & $20(14.6 \%)$ & \\
\hline & $4 \mathrm{~h}$ a wk & $111(5.5 \%)$ & $141(12.6 \%)$ & $69(62.2 \%)$ & $15(13.5 \%)$ & $13(11.7 \%)$ & & $101(91 \%)$ & $10(9 \%)$ & \\
\hline & $>5 \mathrm{~h} \mathrm{a} \mathrm{wk}$ & $262(12.9 \%)$ & $35(13.5 \%)$ & $156(60 \%)$ & $36(13.8 \%)$ & $33(12.7 \%)$ & & $225(85.9 \%)$ & $37(14.1 \%)$ & \\
\hline \multirow{5}{*}{$\begin{array}{c}\text { Duration } \\
\text { of viewing } \\
\text { TV... }\end{array}$} & $<2 \mathrm{~h}$ a day & $541(26.7 \%)$ & $84(15.6 \%)$ & $316(58.5 \%)$ & $79(14.6 \%)$ & $61(11.3 \%)$ & \multirow{5}{*}{0.252} & $475(87.8 \%)$ & $66(12.2 \%)$ & \multirow{5}{*}{0.057} \\
\hline & $2-4$ h a day & $832(41 \%)$ & $114(13.8 \%)$ & $491(59.6 \%)$ & $120(14.6 \%)$ & $99(12 \%)$ & & 757 (91.1\%) & $74(8.9 \%)$ & \\
\hline & & & & & & & & & & \\
\hline & $4-6 \mathrm{~h}$ a day & $349(17.2 \%)$ & $60(17.3 \%)$ & $176(50.9 \%)$ & $53(15.3 \%)$ & $57(16.5 \%)$ & & $300(86 \%)$ & $49(14 \%)$ & \\
\hline & $>8$ h a day & $300(14.8 \%)$ & $43(14.4 \%)$ & $171(57.4 \%)$ & $41(13.8 \%)$ & $43(14.4 \%)$ & & $271(90.6 \%)$ & $28(9.4 \%)$ & \\
\hline \multirow{5}{*}{$\begin{array}{c}\text { Duration } \\
\text { of daylight } \\
\text { sleep }\end{array}$} & Never & $368(18.2 \%)$ & $63(17.3 \%)$ & $194(53.3 \%)$ & $52(14.3 \%)$ & $55(15.1 \%)$ & & $318(86.4 \%)$ & $50(13.6 \%)$ & \multirow{5}{*}{0.252} \\
\hline & $<1 \mathrm{~h}$ & $209(10.3 \%)$ & $35(16.8 \%)$ & $115(55.3 \%)$ & $35(16.8 \%)$ & $23(11.1 \%)$ & & $187(89.5 \%)$ & $22(10.5 \%)$ & \\
\hline & $1-2 h$ & $410(20.2 \%)$ & $55(13.4 \%)$ & $228(55.7 \%)$ & $69(16.9 \%)$ & $57(13.9 \%)$ & 0.129 & $374(91.2 \%)$ & $36(8.8 \%)$ & \\
\hline & $2-3 h$ & $608(30 \%)$ & $95(15.7 \%)$ & $355(58.8 \%)$ & $72(11.9 \%)$ & $82(13.6 \%)$ & & $538(88.5 \%)$ & $70(11.5 \%)$ & \\
\hline & $\geq 4 \mathrm{~h}$ & $422(20.8 \%)$ & $54(12.9 \%)$ & $262(62.4 \%)$ & $62(14.8 \%)$ & $42(10 \%)$ & & 384 (91\%) & $38(9 \%)$ & \\
\hline \multirow{5}{*}{$\begin{array}{c}\text { Duration } \\
\text { of sleep } \\
\text { at night }\end{array}$} & $<4 \mathrm{~h}$ & $197(9.7 \%)$ & $26(13.3 \%)$ & $126(64.3 \%)$ & $20(10.2 \%)$ & $24(12.2 \%)$ & & $176(89.3 \%)$ & $21(10.7 \%)$ & \multirow{5}{*}{0.180} \\
\hline & $4-6 h$ & $527(26 \%)$ & $74(14.2 \%)$ & $288(55.2 \%)$ & $86(16.5 \%)$ & $74(14.2 \%)$ & & $476(90.3 \%)$ & $51(9.7 \%)$ & \\
\hline & $6-8 h$ & $740(36.5 \%)$ & $118(16 \%)$ & $410(55.6 \%)$ & $117(15.9 \%)$ & $92(12.5 \%)$ & 0.380 & $645(87.2 \%)$ & $95(12.8 \%)$ & \\
\hline & $>8 \mathrm{~h}$ & $545(26.9 \%)$ & $83(15.3 \%)$ & $321(59.2 \%)$ & $69(12.7 \%)$ & $69(12.7 \%)$ & & 497 (91.2\%) & $48(8.8 \%)$ & \\
\hline & Total & & $303(15.1 \%)$ & $1157(57.5 \%)$ & $293(14.6 \%)$ & $260(12.9 \%)$ & & $1808(89.3 \%)$ & $217(10.7 \%)$ & \\
\hline
\end{tabular}

Freq. $(\%)=$ Frequency (percentage); $\mathrm{h}=$ hours. 
BMI $(P=0.10)$ nor W_C $(P=0.587)$. Unfortunately, more than half $1058(52.2 \%)$ of students admitted that they were physically inactive, and only 111 (5.5\%) of the participants were physically active for 4 hours per week. Against expectation, there was insignificant association between physical activity and BMI $(P=0.47)$ or W_C $(P=0.116)$.

A total $832(41 \%)$ of respondents watch TV 2 - 4 hours per day, while 300 (14.8\%) out of them spent more than 8 hours watching TV. Nonetheless, results of this study showed that the time spent on TV watching had no significant effect on BMI $(P=0.252)$ or on W_C $(P=0.057)$. Regarding sleeping, $422(20.8 \%)$ of students used to sleep 4 hours or more during the daylight, while only 740 $(36.5 \%)$ of them used to sleep 6 - 8 hours a night. Sleeping time during the daylight or at night had no considerable $(P>0.05)$ effect on both indicators (Table 2).

\subsection{Eating Habits}

The results of this study revealed that 381 (18.8\%) of studied students skipped breakfast, while 875 (43.2\%) of them used to have their breakfast nearly every day. Against expectation, breakfast intake had insignificant effect on BMI ( $P=$ 0.121) and/or W_C. The majority 1591 (78.5\%) of students used to have meals with their families, more than half $893(56.6 \%)$ of them have normal BMI and $215(13.6 \%)$ of them were obese $(P=0.111)$. Only $82(4 \%)$ of respondents did not have fast food meals, while 277 (13.7\%) of them did this on daily basis. Results presented here indicated that fast foods intake had insignificant effects $(P=$ $0.714)$ on BMI and/or W_C $(P=0.137)$. Seldom of investigated students 159 (7.8\%) never had soft drinks during the day, while 649 (32\%) of them had drunk them on daily basis. Soft drinks intake significantly $(P=0.015)$ affects BMI. OW and OB were lower in students skipped diet soft drinks (24.1\%) compared to those consumed these drinks (ranged from $28 \%$ up to $31.6 \%)(P=0.003)$ (Table 3). Table 4 illustrates the effects of eating habits on both BMI and W_C. It was found $425(21 \%)$ of students ate meat every day and $757(37.3 \%)$ of them had meat 1 - 2 times weekly, while only $137(6.8 \%)$ of participants never ate meat. Meat intake had insignificant effects on BMI $(P=0.283)$ or on W_C $(P=0.144)$ (Table 4). Liver intake significantly affected BMI $(P<0.001)$ and on W_C $(P=$ 0.027). A total 7 (18.9\%) of students who ate liver daily were obese, while 93 (11.4\%) of those who never ate liver were obese. About one fifth 393 (19.4\%) of students never included eggs in their meals and 651 (32.1\%) ate 1 - 2 eggs per week, while 110 (5.4\%) of them consumed 5 - 11 eggs weekly. Seafood intake among students was rare; either 1 - 2 times per month 780 (38.5\%) or 293 (14.5\%) out of interviewed students skipped it from their meals. Seafood and eggs intake had insignificant effects on both indicators $(P>0.05)$. Fried foods intake was considered common among students, only 96 (4.7\%) of them never had it through their meals, whereas 871 (43\%) of them ate them 1 - 2 times weekly. Milk intake by schoolchildren was common either daily 693 (34.1\%) or 1 2 times weekly 530 (26.1\%). Although, $57.0 \%$ of lean students consumed milk 
Table 3. Effects of eating habits on BMI and W_C among schoolchildren living in Taif City, KSA.

\begin{tabular}{|c|c|c|c|c|c|c|c|c|c|c|}
\hline & & \multirow[b]{2}{*}{ Freq. (\%) } & \multicolumn{5}{|c|}{ Body mass classification } & \multicolumn{3}{|c|}{ Waist Circumference } \\
\hline & & & Under-weight & $\begin{array}{l}\text { Normal } \\
\text { weight }\end{array}$ & Over-weight & Obesity & $P$-value & No risk & Risk & $P$-value \\
\hline \multirow{5}{*}{$\begin{array}{l}\text { Breakfast } \\
\text { intake }\end{array}$} & Never & $381(18.8 \%)$ & $45(12 \%$ & $227(60.4 \%)$ & $58(12.2 \%)$ & $46(12.2 \%)$ & \multirow{5}{*}{0.121} & $347(91.3 \%)$ & $33(8.7 \%)$ & \multirow{5}{*}{0.426} \\
\hline & $2 \mathrm{a} w \mathrm{wk}$ & $396(19.5 \%)$ & $70(17.7 \%)$ & $225(57 \%)$ & $52(13.2 \%)$ & $48(12.2 \%)$ & & $350(88.6 \%)$ & $45(11.4 \%)$ & \\
\hline & $3 \mathrm{a} \mathrm{wk}$ & $174(8.6 \%)$ & $17(9.8 \%)$ & $94(54 \%)$ & $33(19 \%)$ & $30(17.2 \%)$ & & $150(86.2 \%)$ & $24(13.8 \%)$ & \\
\hline & $4 \mathrm{a} w \mathrm{wk}$ & $186(19.2 \%)$ & $35(18.8 \%)$ & $96(51.9 \%)$ & $31(16.8 \%)$ & $23(12.4 \%)$ & & $170(91.4 \%)$ & $16(8.6 \%)$ & \\
\hline & $\geq 5$ a wk & $875(43.2 \%)$ & $134(15.4 \%)$ & $505(58.2 \%)$ & $119(13.7 \%)$ & $110(12.7 \%)$ & & $777(88.8 \%)$ & $98(11.2 \%)$ & \\
\hline \multirow{5}{*}{$\begin{array}{l}\text { Light meal } \\
\text { intake }\end{array}$} & Daily & $846(41.7 \%)$ & $126(15 \%)$ & $498(95.4 \%)$ & $121(14.4 \%)$ & $94(11.2 \%)$ & \multirow{5}{*}{0.269} & $765(89.4 \%)$ & $90(10.6 \%)$ & \multirow{5}{*}{0.953} \\
\hline & $4-6$ a wk & $301(14.8 \%)$ & $43(14.3 \%)$ & $170(56.7 \%)$ & $46(15.3 \%)$ & $41(13.7 \%)$ & & $268(89.3 \%)$ & $32(10.7 \%)$ & \\
\hline & $2-3 a$ wk & $468(23.1 \%)$ & $78(16.8 \%)$ & $249(53.7 \%)$ & $72(15.5 \%)$ & $65(14 \%)$ & & $416(88.9 \%)$ & $52(11.1 \%)$ & \\
\hline & $1-2$ a wk & $182(9 \%)$ & $33(18.2 \%)$ & $107(59.1 \%)$ & $20(11 \%)$ & $21(11.6 \%)$ & & $165(90.7 \%)$ & $17(9.3 \%)$ & \\
\hline & never & $212(10.5 \%)$ & $20(9.5 \%)$ & $123(58.3 \%)$ & $33(15.6 \%)$ & $35(16.6 \%)$ & & $187(88.2 \%)$ & $25(11.8 \%)$ & \\
\hline \multirow{4}{*}{$\begin{array}{l}\text { Eating } \\
\text { with } \\
\text { family }\end{array}$} & Daily & $1591(78.5 \%)$ & $244(15.5 \%)$ & 893 (56.66\%) & $227(14.4 \%)$ & $215(13.6 \%)$ & \multirow{4}{*}{0.111} & $1420(89.4 \%)$ & $169(10.6 \%)$ & \multirow{4}{*}{0.05} \\
\hline & $3-4$ & $76(3.7 \%)$ & $13(17.3 \%)$ & $44(58.7 \%)$ & $13(17.3 \%)$ & $5(6.7 \%)$ & & $70(92.1 \%)$ & $6(7.9 \%)$ & \\
\hline & $1-2$ & $194(9.6 \%)$ & $32(16.5 \%)$ & $114(58.8 \%)$ & $22(11.3 \%)$ & $26(13.4 \%)$ & & $164(84.5 \%)$ & $30(15.5 \%)$ & \\
\hline & Rarely & $163(8 \%)$ & $14(8.6 \%)$ & $103(63.6 \%)$ & $31(19.1 \%)$ & $14(8.6 \%)$ & & $152(93.3 \%)$ & $11(6.7 \%)$ & \\
\hline \multirow{5}{*}{$\begin{array}{l}\text { Fast food } \\
\text { intake }\end{array}$} & Daily & $277(13.7 \%)$ & $42(15.6 \%)$ & $146(54.1 \%)$ & $42(15.6 \%)$ & $40(14.8 \%)$ & \multirow{5}{*}{0.714} & $252(91.3 \%)$ & $24(8.7 \%)$ & \multirow{5}{*}{0.137} \\
\hline & $4-6$ a wk & $560(27.6 \%)$ & $84(15.1 \%)$ & $232(59.7 \%)$ & $76(13.7 \%)$ & $64(11.5 \%)$ & & $501(89.5 \%)$ & $59(10.5 \%)$ & \\
\hline & $2-3 \mathrm{awk}$ & $667(32.9 \%)$ & $104(15.6 \%)$ & 387 (58.2\%) & $89(13.4 \%)$ & $85(12.8 \%)$ & & $599(89.8 \%)$ & $68(10.2 \%)$ & \\
\hline & $1-2$ a wk & $432(21.3 \%)$ & $63(14.6 \%)$ & $240(55.7 \%)$ & $68(15.8 \%)$ & $60(13.9 \%)$ & & $373(86.3 \%)$ & $59(13.7 \%)$ & \\
\hline & never & $82(4 \%)$ & $8(9.8 \%)$ & $46(56.1 \%)$ & $18(22 \%)$ & $10(12.2 \%)$ & & $76(93.8 \%)$ & $5(6.2 \%)$ & \\
\hline \multirow{5}{*}{$\begin{array}{l}\text { Soft drink } \\
\text { intake }\end{array}$} & Daily & $649(32 \%)$ & $98(15.3 \%)$ & $354(55.2 \%)$ & $86(13.4 \%)$ & $103(16.12 \%)$ & \multirow{5}{*}{0.015} & $573(88.4 \%)$ & 75 (11.6\%) & \multirow{5}{*}{0.707} \\
\hline & $4-6$ a wk & $506(25 \%)$ & $88(17.4 \%)$ & $293(57.9 \%)$ & $76(15 \%)$ & $49(9.7 \%)$ & & 454 (89.9\%) & $51(10.11 \%)$ & \\
\hline & $2-3 a$ wk & $433(21.4 \%)$ & $61(14.2 \%)$ & $250(58.1 \%)$ & $59(13.7 \%)$ & $60(14 \%)$ & & $384(88.7 \%)$ & $49(113 \%)$ & \\
\hline & $1-2 \mathrm{a} \mathrm{wk}$ & $276(13.6 \%)$ & $30(11 \%)$ & $158(57.9 \%)$ & $51(18.7 \%)$ & $34(12.5 \%)$ & & $284(89.9 \%)$ & $28(10.1 \%)$ & \\
\hline & never & $159(7.8 \%)$ & $25(15.7 \%)$ & $101(63.5 \%)$ & $21(13.2 \%)$ & $12(7.5 \%)$ & & $146(91.8 \%)$ & $13(8.2 \%)$ & \\
\hline \multirow{5}{*}{$\begin{array}{c}\text { Diet soft } \\
\text { drink }\end{array}$} & Daily & $171(8.4 \%)$ & $29(17.1 \%)$ & $92(54.8 \%)$ & $21(12.5 \%)$ & $26(15.5 \%)$ & \multirow{5}{*}{0.003} & $184(87.1 \%)$ & $22(12.9 \%)$ & \multirow{5}{*}{0.773} \\
\hline & $4-6$ a wk & $190(9.4 \%)$ & $25(13.2 \%)$ & $105(55.3 \%)$ & $34(17.9 \%)$ & $26(13.7 \%)$ & & $167(88.4 \%)$ & $22(11.6 \%)$ & \\
\hline & $2-3 a w k$ & $226(11.1 \%)$ & $31(13.8 \%)$ & $125(55.8 \%)$ & $32(14.3 \%)$ & $36(16.1 \%)$ & & $205(90.7 \%)$ & $21(9.3 \%)$ & \\
\hline & $1-2 \mathrm{a} w \mathrm{k}$ & $266(13.1 \%)$ & $32(12.2 \%)$ & $135(51.3 \%)$ & $41(15.6 \%)$ & $55(20.9 \%)$ & & $235(88.3 \%)$ & $31(11.7 \%)$ & \\
\hline & never & $1169(57.7 \%)$ & $184(15.8 \%)$ & $699(60.1 \%)$ & $164(14.1 \%)$ & $116(10 \%)$ & & 1049 (87.7\%) & $120(10.3 \%)$ & \\
\hline \multirow{5}{*}{$\begin{array}{c}\text { High } \\
\text { calorie } \\
\text { beverage }\end{array}$} & Daily & $150(7.4 \%)$ & $23(15.5 \%)$ & $88(59.5 \%)$ & $17(11.5 \%)$ & $20(13.5 \%)$ & \multirow{5}{*}{0.473} & $140(94 \%)$ & $9(6 \%)$ & \multirow{5}{*}{0.187} \\
\hline & $4-6$ a wk & $225(11.1 \%)$ & $31(14 \%)$ & $125(56.3 \%)$ & $38(17.1 \%)$ & $28(12.3 \%)$ & & $205(91.1 \%)$ & $20(8.9 \%)$ & \\
\hline & $2-3 a$ wk & $296(14.6 \%)$ & $37(12.5 \%)$ & $165(55.9 \%)$ & $4(15.9 \%)$ & $46(15.6 \%)$ & & $261(88.2 \%)$ & $35(11.8 \%)$ & \\
\hline & $1-2 \mathrm{a} w \mathrm{wk}$ & $472(23.3 \%)$ & $79(16.9 \%)$ & $265(56.6 \%)$ & $57(12.2 \%)$ & 67 (14.3\%) & & $410(87 \%)$ & $61(13 \%)$ & \\
\hline & never & $878(43.3 \%)$ & $132(15.1 \%)$ & $512(58.6 \%)$ & $133(15.2 \%)$ & 97 (11.1\%) & & $787(89.6 \%)$ & $91(10.4 \%)$ & \\
\hline \multicolumn{3}{|l|}{ Total } & $303(15.1 \%)$ & $1157(57.5 \%)$ & $293(14.6 \%)$ & $26(12.9 \%)$ & & $1808(89.3 \%)$ & $217(10.7 \%)$ & \\
\hline
\end{tabular}

Freq. $(\%)=$ Frequency (percentage); wk = week. 
Table 4. Effects of eating habits on body mass index and waist circumference.

\begin{tabular}{|c|c|c|c|c|c|c|c|c|c|c|}
\hline & & \multirow{2}{*}{ Freq. (\%) } & \multicolumn{5}{|c|}{ Body mass classification } & \multicolumn{3}{|c|}{ Waist Circumference } \\
\hline & & & Under-wt & Normal-wt & Over-wt & OB & $P$-value & No risk & Risk & $P$-value \\
\hline \multirow{5}{*}{$\begin{array}{l}\text { Meat } \\
\text { intake }\end{array}$} & Daily & $425(21 \%)$ & $65(15.4 \%)$ & $234(55.3 \%)$ & $63(14.9)$ & $61(14 \%)$ & \multirow{5}{*}{0.283} & $381(89.9 \%)$ & $43(10.1 \%)$ & \multirow{5}{*}{0.144} \\
\hline & $1-2 \mathrm{awk}$ & $757(37.3 \%)$ & $108(14.4 \%)$ & $447(59.5 \%)$ & $106(14.1 \%)$ & $90(12 \%)$ & & $676(89.3 \%)$ & $81(10.7 \%)$ & \\
\hline & $3-4$ awk & $495(24.4 \%)$ & $87(17.7 \%$ & $266(54.1 \%)$ & $67(13.6 \%)$ & $72(14.6 \%)$ & & $430(86.9 \%)$ & $65(13.1 \%)$ & \\
\hline & $5-6$ awk & $209(10.3 \%)$ & $29(14 \%)$ & $116(56 \%)$ & $38(18.4 \%)$ & $24(11.6 \%)$ & & $187(89.9 \%)$ & $21(10.1 \%)$ & \\
\hline & Never & $137(6.8 \%)$ & $14(10.3 \%)$ & $92(67.6 \%)$ & $18(13.2 \%)$ & $12(8.8 \%$ & & $130(94.9 \%)$ & $7(5.1 \%)$ & \\
\hline \multirow{5}{*}{$\begin{array}{l}\text { Liver in- } \\
\text { take }\end{array}$} & Daily & $37(1.8 \%)$ & $6(16.2 \%)$ & $19(51.4 \%)$ & $5(13.5 \%)$ & $7(18.9 \%)$ & \multirow{5}{*}{$<0.001$} & $34(91.9 \%)$ & $3(8.1 \%)$ & \multirow{5}{*}{0.027} \\
\hline & $1-2 \mathrm{awk}$ & $780(38.5 \%)$ & $142(18.3 \%)$ & $419(53.9 \%)$ & $107(13.8 \%)$ & $109(14 \%)$ & & $673(86.4 \%)$ & $106(13.6 \%)$ & \\
\hline & $3-4$ awk & $193(9.5 \%)$ & $34(18 \%)$ & $109(57.7 \%)$ & $27(14.3)$ & $19(10.1 \%)$ & & $172(89.1 \%)$ & $21(10.9 \%)$ & \\
\hline & $5-6$ awk & $190(9.4 \%)$ & $28(14.8 \%)$ & $99(52.4 \%)$ & $35(18.5 \%)$ & $27(14.3 \%)$ & & $170(89.9 \%)$ & $19(10.1 \%)$ & \\
\hline & Never & $820(40.5 \%)$ & $93(11.4 \%)$ & $510(62.7 \%)$ & $118(14.5 \%)$ & $93(11.4 \%)$ & & 753 (91.8\%) & $67(8.2 \%)$ & \\
\hline \multirow{6}{*}{$\begin{array}{c}\text { Egg } \\
\text { intake }\end{array}$} & $\geq 12$ awk & $164(8.1 \%)$ & $30(18.3 \%)$ & $90(54.9 \%)$ & $17(10.4 \%)$ & $27(16.5 \%$ & \multirow{6}{*}{0.084} & 147 (9.6\%) & $17(10.4 \%)$ & \multirow{6}{*}{0.24} \\
\hline & $8-11 w k$ & $110(5.4 \%)$ & $14(12.8 \%)$ & $56(51.4 \%)$ & $26(23.9 \%)$ & $13(11.9 \%)$ & & $96(87.3 \%)$ & $14(12.7 \%)$ & \\
\hline & 5 - 7 awk & 237 (11.7\%) & 33 (13.9\%) & $140(59.1 \%)$ & $34(14.3 \%)$ & $30(12.7)$ & & $207(87.3 \%)$ & $30(12.7 \%)$ & \\
\hline & $2-4$ awk & 465 (22.9\%) & $70(15.2 \%)$ & $269(58.2 \%)$ & $59(12.8 \%)$ & $64(13.9 \%)$ & & 403 (86.9\%) & $61(13.1 \%)$ & \\
\hline & $<2$ awk & $651(32.1 \%)$ & $93(14.4 \%)$ & $373(57.8 \%)$ & 95 (14.7\%) & $84(13 \%)$ & & $594(91.2 \%)$ & $57(8.8 \%)$ & \\
\hline & Never & $393(19.4 \%)$ & $59(15.2 \%)$ & 227 (584\%) & $62(15.9 \%)$ & $41(105 \%)$ & & 355 (90.6\%) & $37(9.4 \%)$ & \\
\hline \multirow{5}{*}{$\begin{array}{c}\text { Fish \& } \\
\text { seafood } \\
\text { intake }\end{array}$} & $1-2$ month & $780(38.5 \%)$ & $115(14.8 \%)$ & 441 (56.9\%) & $120(15.5 \%)$ & $99(12.8 \%)$ & & $687(88.2 \%)$ & $92(11.8 \%)$ & \multirow{5}{*}{0.573} \\
\hline & 3 - 4 month & 464 (22.9\%) & $73(15.9 \%)$ & $260(56.5 \%)$ & $62(13.5 \%)$ & $65(14.1 \%)$ & & $412(88.8 \%)$ & $52(11.2 \%)$ & \\
\hline & $5-6$ a month & 197 (9.7\%) & $27(13.8 \%)$ & $121(61.7 \%)$ & $23(11.7 \%)$ & $25(12.8 \%)$ & 0.629 & $178(90.4 \%)$ & $19(9.6 \%)$ & \\
\hline & $>6$ a mo & $282(13.9 \%)$ & $36(12.8 \%)$ & $169(60.1 \%)$ & 37 (13.2\%) & 39 (13.9\%) & & 257 (91.5\%) & $24(8.5)$ & \\
\hline & Never & $293(14.5 \%)$ & $84(16.6 \%)$ & $161(55.5 \%)$ & $50(17.2 \%)$ & $31(10.7)$ & & $265(90.4 \%)$ & $28(9.6 \%)$ & \\
\hline \multirow{5}{*}{$\begin{array}{c}\text { Fried food } \\
\text { intake }\end{array}$} & Daily & $294(14.5 \%)$ & 37 (12.7\%) & $17(61.2 \%)$ & $34(11.7 \%)$ & $42(14.4 \%)$ & & $273(92.9 \%)$ & $21(7.1 \%)$ & \multirow{5}{*}{0.257} \\
\hline & $1-2$ awk & $871(43 \%)$ & $126(14.5 \%)$ & $498(57.5)$ & $124(14.3 \%)$ & $118(13.6 \%)$ & & $769(88.5 \%)$ & $100(11.5 \%)$ & \\
\hline & 3 - 4 awk & $535(26.4 \%)$ & $92(17.3 \%)$ & $311(58.3 \%)$ & $75(14.1 \%)$ & $55(10.3 \%)$ & 0.009 & $481(89.9 \%)$ & $54(10.1 \%)$ & \\
\hline & $5-6$ awk & $212(10.5 \%)$ & $30(14.4 \%)$ & $112(53.8 \%)$ & $42(20.2 \%)$ & $24(11.5 \%)$ & & $185(87.3 \%)$ & $27(12.7 \%)$ & \\
\hline & Never & $96(4.7 \%)$ & 10 (10.4\%) & 50 (52.1\%) & 17 (17.7\%) & $19(19.8 \%)$ & & 84 (87.5\%) & $12(12.5 \%)$ & \\
\hline
\end{tabular}

Freq. $(\%)=$ Frequency (percentage); awk = a week.

on daily basis compared to $14.7 \%$ of OW and $12.5 \%$ of obese children, the difference was statistically insignificant $(\mathrm{p}=0.732)$. Vegetables intake was uncommon since 945 (46.6\%) of participants had them 1 - 2 times per week or 234 (11.5\%) never ate vegetables in their meals (Table 5). 
Table 5. Effects of eating dairy products, vegetables and grains habits on body mass index and waist circumference.

\begin{tabular}{|c|c|c|c|c|c|c|c|c|c|c|}
\hline & & \multirow{2}{*}{ Freq. (\%) } & \multicolumn{5}{|c|}{ Body mass classification } & \multicolumn{3}{|c|}{ Waist Circumference } \\
\hline & & & Under-weight & $\begin{array}{c}\text { Normal } \\
\text { weight }\end{array}$ & Over-weight & OB & $P$-value & No risk & Risk & $P$-value \\
\hline \multirow{5}{*}{ Milk intake } & Daily & $691(34.1 \%)$ & $108(15.7 \%)$ & $391(57.0 \%)$ & $101(14.7 \%)$ & $86(12.5 \%)$ & \multirow{5}{*}{0.732} & $621(90 \%)$ & $69(10 \%)$ & \multirow{5}{*}{0.256} \\
\hline & $1-2$ awk & $530(26.1 \%)$ & $79(15.0 \%)$ & $309(58.5 \%)$ & $75(14.2 \%)$ & $65(12.3 \%)$ & & $472(89.2 \%)$ & $57(10.8 \%)$ & \\
\hline & $3-4$ awk & $332(16.4 \%)$ & $48(14.5 \%)$ & $184(55.6 \%)$ & $51(15.4 \%)$ & $48(14.5 \%)$ & & $287(86.4 \%)$ & $45(13.6 \%)$ & \\
\hline & $5-6$ awk & $199(9.8 \%)$ & $30(15.1 \%)$ & $105(52.8 \%)$ & $36(18.1 \%)$ & $28(14.1 \%)$ & & $176(88.4 \%)$ & $23(11.6 \%)$ & \\
\hline & Never & $265(13.1 \%)$ & $35(13.5 \%)$ & $163(62.9 \%)$ & $30(11.6 \%)$ & $31(12.0 \%)$ & & $244(92.1 \%)$ & $21(7.9 \%)$ & \\
\hline \multirow{4}{*}{$\begin{array}{l}\text { Vegetables } \\
\text { intake per day }\end{array}$} & $\geq 5$ & $429(21.2 \%)$ & $51(12 \%)$ & $264(62.0 \%)$ & $58(13.6 \%)$ & $53(12.4 \%)$ & \multirow{4}{*}{0.424} & $395(92.1 \%)$ & $34(7.9 \%)$ & \multirow{4}{*}{0.012} \\
\hline & $3-4$ & $409(20.2 \%)$ & $70(17.2 \%)$ & $215(52.7 \%)$ & $66(16.2 \%)$ & $57(14 \%)$ & & $364(89.2 \%)$ & $44(10.8 \%)$ & \\
\hline & $1-2$ & $945(46.6 \%)$ & $152(16.2 \%)$ & $538(57.4 \%)$ & $132(14.1 \%)$ & $115(12.3 \%)$ & & $838(88.8 \%)$ & $106(11.2 \%)$ & \\
\hline & Never & $234(11.5 \%)$ & $28(12.1 \%)$ & $135(58.2 \%)$ & $35(15.1 \%)$ & $34(14.7 \%)$ & & $205(87.6 \%)$ & $29(12.4 \%)$ & \\
\hline \multirow{4}{*}{$\begin{array}{c}\text { Consumption } \\
\text { of grains per } \\
\text { day }\end{array}$} & $\geq 5$ & $473(23.3 \%)$ & $64(13.6 \%)$ & $284(60.4 \%)$ & $64(13.6 \%)$ & $58(12.3 \%)$ & \multirow{4}{*}{0.293} & 437 (92.4\%) & $36(7.6 \%)$ & \multirow{4}{*}{0.002} \\
\hline & $3-4$ & 447 (22.1\%) & $72(16.2 \%)$ & $255(57.3 \%)$ & $68(15.3 \%)$ & $50(11.2 \%)$ & & 409 (91.5\%) & 38 (8.5\%) & \\
\hline & $1-2$ & 958 (47.3\%) & $146(15.4 \%)$ & $536(56.4 \%)$ & $140(14.7 \%)$ & $128(13.5 \%)$ & & $825(86.3 \%)$ & $131(13.7 \%)$ & \\
\hline & Never & 137 (6.8\%) & $16(11.8 \%)$ & 77 (56.6\%) & $21(15.4 \%)$ & $22(16.2 \%)$ & & 125 (91.2\%) & $12(8.8 \%)$ & \\
\hline
\end{tabular}

Freq. $(\%)=$ Frequency (percentage); awk = a week.

\section{Discussion}

The prevalence of OW+ OB was $27.5 \%$. Similar results were reported from different areas of KSA. For example, AL-Oboudi [12] reported that the combined prevalence of OW and OB (26\%) among school girls in Riyadh. In the eastern Province, Abahussain [6] showed that $28 \%$ of school girls were OW and obese. El-Hazmi and Warsy [10] showed that the highest percentage of OW + OB was reported in the Eastern Province of Saudi Arabia [10]. Farghly et al. [11] found that the combined OW and OB (26.9\%) in a Abha area (high altitude city similar to Taif City), which was very close to our figure (27.5\%). Recently, higher rates of OW (19.5\%) and OB (24.1\%) were reported among secondary male students in three main cities of KSA [19].

Although BMI is the most common indicator of $\mathrm{OB}$ worldwide, but recently body fat percent could be considered as a more desirable indicator of $\mathrm{OB}$ than BMI [7]. Results presented here showed that $27.5 \%$ of participants were obese or overweight (i.e. one in every four schoolchildren is obese or overweight, while 20 years earlier, Al-Nuaim et al. [20] reported that one in every 6 children were obese).

There were significant associations $(P<0.001)$ between participants' gender and their BMI classification, $31.9 \%$ of males and only $21.9 \%$ of females were overweight and obese. This may could be due to awareness of female students 
toward their body appearance than males [11], and boys and girls are different with regard their lifestyle. Similar results were reported by Al-Hazzaa et al. [19]. Results of this study are inconsistent with those of Farghly et al. [11] who found higher rates among female schoolchildren (29\%) than males (23.9\%) in Abha area. Waist circumference presented in this study showed that $15.8 \%$ of boys were at risk of developing health problems compared to only $4.3 \%$ of girls. Our results disagreed with those of Al-Hazzaa et al. [19] who found that the mean W_C of school girls in three major cities in KSA was higher than males.

Results presented here showed that socio-economic status had an effect on rates of $\mathrm{OW}$ and $\mathrm{OB}$, which agreed with those of Amin et al. [8] and Al-Hazzaa et al. [19]. On the other hand, our results are inconsistent with Katzmarzyk [21] who cited that Canadian children from low income families have higher rates of OW compared to their counterparts in high income families.

Results showed that location of residency had a significant $(P<0.001)$ effect on BMI. Southern part of Taif City is highly populated compared to other areas, it is the place where a significant number of restaurants and fast food outlets are located. These factors might be behind high prevalence of OW + OB. However, further study should be conducted to investigate effects of location of residency on rates of $\mathrm{OW}$ and $\mathrm{OB}$.

Smoking in the current study neither affected BMI $(P=0.10)$ nor W_C $(P=$ 0.587). Farghly et al. [11] found $8.6 \%$ of male intermediate schoolchildren in Abha smoke.

It is well known that physical activity is of great significance for normal growth and development of healthy children and youths. Moderate level of physical activities when the heart rate exceeds 139 beat per minute, while the vigorous one when the heart rate tops 159 beat per minute [22]. American Heart Association recommends that these two levels to be conducted by children and youths for at least 1 hour a day [22]. Physical inactivity is considered a major coronary artery disorder risk factor. Furthermore, it also raises the risk of stroke and other main CVD, such as adiposity, high blood pressure, low level of good cholesterol (HDL-c) and diabetes mellitus [22]. More than half of participants were physically inactive. Recent published data showed that majority of children $(60 \%)$ and $71 \%$ of Saudi adolescents were physically inactive [22]. Omer et al. [2] reported much higher rates of physical inactivity (91.7\%) among schoolchildren. Collison et al. [5] found that frequency of physical activity decreased as the age increases in both sexes. There are many reasons for high rate of physical inactivity, including dependence of children and adolescents on cars for daily activities, high rates of air pollution and high temperatures especially during summer times discourage performing outdoor activities. It was shown that levels of physical activities were lower and levels of TV viewing were higher among OW youths than lean youths aged 10 - 16 years of 34 countries worldwide [23]. Additionally, physical activity not only affects rates of OW and OB, but it was found that it affects blood lipid profile, For example, AL-Hazzaa [22] found that inactive Saudi boys have unfavorable blood lipid profile compared to active boys. 
Results showed that the time spent on TV watching had insignificant effect on BMI $(P=0.252)$ or W_C $(P=0.057)$. Similar effect was reported by Omer et al. [2]. In Abha region, schoolchildren were not occupied with TV watching or playing video [11].

Sleeping during the daylight or at night had no considerable effect on both indicators $(P>0.05)$. Our results contradicted those of Collison et al. [5] who found negative relationship between BMI and W_C and hours of night sleep among boys, while among girls only sleep at night had an impact on both anthropometric indicators.

Many reasons are behind considering breakfast as the most important meal during the day. It provides schoolchildren with a sufficient amount of energy for appropriate brain functions, thus, it improves learning skills. Without it, energy reserves would be depleted overnight, thus, it resulted in a decline in the level of blood glucose. If this decrease is considerable, it disturbs functions of cerebral [12]. Furthermore, Niklas and his colleagues [24] argued that eating breakfast at regular basis could control body mass, possibly due to low fat diet and lower consumption of high caloric snacks. Breakfast intake had insignificant effect on BMI $(P=0.121)$ and W_C, which agrees with those of Omer et al. [2], and with those of Al-Rethaiaa et al. [7]. Abalkhail and Shawky [25] found that almost 15\% of schoolchildren in Jeddah City skipped their breakfast. Similarly, $16.5 \%$ of school girls in Riyadh skipped their breakfast [26]. Amin et al. [8] indicated that $47.8 \%$ of obese and overweight male primary schoolchildren in Al-Hassa (KSA) skipped their breakfast. Farghly et al. [11] found that $28 \%$ of schoolchildren in Abha area skipped their breakfast, and it was more alarming as $17 \%$ of female high school students never ate breakfast. Results of this study disagreed with others. For example, Al-Aboudi [12] found that obese females were more likely to skip breakfast. Several reasons could be behind high rate of skipping breakfast in this study; in KSA, the school day begins too early, thus, the schoolchildren could not have enough time or appetite to have their breakfast [11] and it is well known that teenagers are skipping their breakfast at higher rates compared to other age groups.

Results of this study support initial assumption that eating with a family has an impact on BMI and W_C. It is a rational to propose that frequent consumption of meals with a family would be associated with low prevalence of OW/OB. Many probable mechanisms were cited by Utter and his colleagues [27] for the positive relationships between eating with a family and nutrition of the youths, such as availability of healthy food options, constructive family debate regarding food and nutrition, and/or parental influence on eating habits. Amin et al. [8] indicated a positive link between food intake away from family and BMI. Results presented in this study contradicted findings of Al-Rethaiaa et al. [7].

Avoiding or minimizing fast foods intake is a healthy practice that needs to be encouraged for the following reasons; firstly, the nutritional value of fast foods is questionable; secondly, children may select unbalanced fast foods; thirdly, fast foods are deficient in many essential elements, have high caloric intake, and high 
contents of fats and sodium. This could be explained via the fact that majority of the participants are living with their families, thus, families play a crucial role in affecting children eating habits and food choices. Collison et al. [5] showed that schoolchildren in Riyadh consumed at an average of 4.5 fast food meals every week.

One probable explanation of positive link between soft drinks intake and BMI could be that consumption of excessive sugars deposited as fat in the body, leading to body weight increase and, thus, increased OB rate [28]. Furthermore, diets with high sugars have contributed to the development of metabolic dyslipidemia and insulin resistance diabetes [29]. Collison et al. [5] showed positive link between uptake of sugary drinks and both indicators (BMI and W_C). In a large cross-sectional study ( 24,000 US citizens) indicated that $19 \%$ of overweight and $22 \%$ of obese participants consume diet beverages [30]. It also found that obese and overweight adults who drink diet drinks tended to have more food [30]. Researches of this study offered two possible hypothesis; sugar-free drinks still stimulate the mind's "sugar reward" pathways, thus, it causes the person to snack more since he has a "sweet tooth" [30]; and the person might simply transfer the energy uptake he used to have from beverages to increase food consumption [30].

There is an increasing evidence that, an increase in dairy consumption by almost 2 serving a day could decrease the risk of being OW by a percent up to 70\% [31]. Furthermore, high intake of calcium every day was found to be related to reduce adiposity among children that have been investigated longitudinally [31]. Another health benefit of higher calcium uptake was shown in a study conducted by Pereira et al. [32]. Farghly et al. [11] found that 51.5\% of schoolchildren in Abha (KSA) consumed milk on daily basis. The same trend was noted in this study results when considering W_C. Amin et al. [8] noted that overweight and obese boys consumed less milk and dairy products. While Collison et al. [5] found milk intake by schoolchildren inversely associated with BMI and W_C.

It is well known that fruits and vegetables play a crucial role in reducing overall caloric intake, since their contents of water and fiber are high. Therefore, adding fruits and vegetables to meals is beneficial in weight management. In the present study vegetables intake was uncommon. This could be explained as; in Saudi society, consumption of raw fruits and vegetables during the course of a meal is not common, and the fruits and vegetables constituents in majority of the Saudi meals are too little to affect the overall energy intake [7], also most of Saudis eat fruits at the end of their meals as treats, therefore, resulting in losing their "satiety impact" that decreases the overall caloric intake of the diet [7]. In the present study, when W_C was used to assess nutritional status, it was found that $92.1 \%$ of participants were at least health risk linked with excess abdominal lipids since they consumed 5 or more servings of fruits and vegetables per day. Amin and his team [8] found that overweight and obese schoolchildren consumed less serving of fresh fruits (26.5\% vs. $41.4 \%$ in lean) and vegetables $(36.8 \%$ vs. $39.4 \%$ ) compared to their normal fellows. 


\section{Conclusions}

The current study revealed that prevalence of combined OW and OB was $27.5 \%$. It was significantly higher among boys than girls (31.9\% vs. $21.9 \%)$, and among schoolchildren from high income families. Smoking of a family member and stress have considerably related to W_C. Unfortunately, more than half $52.2 \%$ of students were physically inactive. Regular soft or diet drinks had significant effects on BMI; the other investigated eating habits had no such effects on BMI. The association between liver intake and BMI and W_C was statistically significant. Furthermore, results showed considerable link between consumption of fruits/vegetables and grains and W_C. The prevalence of OW and OB reported in this study and other studies in the kingdom showed the prevalence of OW and $\mathrm{OB}$ approaching or even exceeding rates in several developed countries. OW and $\mathrm{OB}$ were the main prevalent nutritional problem among schoolchildren in Taif area, an overall prevalence (27.5\%) is very alarming. Reasons for poor nutritional status among schoolchildren in KSA are: poor eating habits, namely skipping breakfast, low consumption of fruits and vegetables, low milk uptake, increase consumption of soft drinks and high-caloric drinks. Thus, an intervention national wide program is urgent to revert or decrease the increasing rates of $\mathrm{OW}$ and $\mathrm{OB}$ and the probable health hazards which could result in grave consequences on adult populations and health care system.

Limitation of this study could be attributed to precision of the self-reported data concerning dietary intake and lifestyle of the participants. This is an attribute shared by several studies of the same nature (cross-sectional).

\section{Ethical Consideration}

This study had obtained ethical approval (No: 83056) from the Ethical Committee at Taif University, KSA. Informed verbal consent was obtained from every student before filling the study tool.

\section{References}

[1] General Census of Population and Housing [Internet]. Riyadh: Central Department of Statistics and Information. http://www.stats.gov.sa/en

[2] Omer, E.O.M., Al Sheri, M.A. and Al Bakri, U.M. (2013) Nutritional Status of Public Elementary School Boys in Al-Baha City, Saudi Arabia. Journal of Nutrition \& Food Sciences, 3, 1-5.

[3] Bray, G.A. (2002) Predicting Obesity in Adults from Childhood and Adolescent Weight. American Society for Clinical Nutrition, 76, 497-498.

[4] Ebbeling, C.B., Pawlak, D.B. and Luwig, D.S. (2002) Childhood Obesity: PublicHealth Crisis, Common Sense Cure. The Lancet, 360, 473-482.

https://doi.org/10.1016/S0140-6736(02)09678-2

[5] Collison, K.S., Zaidi,M.Z., Subhani, S.N., Al-Rubeaan, K., Shoukri, M. and Al-Mohanna, F.A. (2010) Sugar-Sweetened Carbonated Beverage Consumption Correlates with BMI, Waist Circumference, and Poor Dietary Choices in School Children. BMC Public Health, 10, 1-13. https://doi.org/10.1186/1471-2458-10-234

[6] Abahussain, N.A. (1999) Nutritional Status of Adolescent Girls in the Eastern Province 
of Saudi Arabia. Nutrition and Health, 13, 171-177. https://doi.org/10.1177/026010609901300305

[7] Al-Rethaiaa, A.S., Fahmy, A.-E. and Al-Shwaiyat, N. M. (2010) Obesity and Eating Habits among College Students in Saudi Arabia: A Cross Sectional Study. Nutrition Journal, 9, 39-48. https://doi.org/10.1186/1475-2891-9-39

[8] Amin, T.T., A-Sultan, A.I. and Ali, A. (2008) Overweight and Obesity and Their Relation to Dietary Habits and Socio-Demographic Characteristics among Male Primary School Children in Al-Hassa, Kingdom of Saudi Arabia. European Journal of Nutrition, 47, 310-318. https://doi.org/10.1007/s00394-008-0727-6

[9] El-Qudah, J.M., Al-Omran, H., Abu-Alsoud, B. and Al-Shek Yousef, T.O.I. (2012) Nutritional Status among a Sample of Saudi College Students. Current Research Journal of Biological Sciences, 4, 557-562.

[10] El-Hazmi, M.A.F. and Warsy, A.S. (2002) The Prevalence of Obesity and Overweight in 1 - 18-Year-Old Saudi Children. Annals of Saudi Medicine, 22, 303-307.

[11] Farghaly, N.F., Ghazali, B.M., Al-Wabel, H.M., Sadek, A.A. and Abbag, F.I. (2007) Life Style and Nutrition and Their Impact on Health of Saudi School Students in Abha, Southwestern Region of Saudi Arabia. Saudi Medical Journal, 28, 415-421.

[12] Al-Oboudi, L.M. (2010) Impact of Breakfast Eating Pattern on Nutritional Status, Glucose Level, Iron Status in Blood and Test Grades among Upper Primary School Girls in Riyadh City, Saudi Arabia. Pakistan Journal of Nutrition, 9, 106-111. https://doi.org/10.3923/pjn.2010.106.111

[13] Almuhanna, M.A., Alsaif, M., Alsaadi, M. and Almajwal, A. (2014) Fast Food Intake and Prevalence of Obesity in School Children in Riyadh City. Sudan Journal of Paediatric, 14, 71-80.

[14] Abahussain, N.A. (2011) Was There a Change in the Body Mass Index of Saudi Adolescent Girls in Al-Khobar between 1997 and 2007? Journal of Family and Community Medicine, 18, 49-53. https://doi.org/10.4103/2230-8229.83367

[15] Al-Shehri, J.A. (2014) Childhood Obesity Prevalence among Primary Schooboys at Al-Iskan Sector, Holly Makkah, Suadi Arabia. International Journal of Medical Science and Public Health, 3, 150-154. https://doi.org/10.5455/ijmsph.2013.061120131

[16] Kuczmarski, R.J., Ogden, C.L., Guo, S.S., et al. (2002) 2000 CDC Growth Charts for the United States: Methods and Development. National Center for Health Statistics. Vital Health Stat 11.

[17] Goonasegaran, A.R., Nabila, F. and Shuhada, N.S. (2012) Comparison of the Effectiveness of Body Mass Index and Body Fat Percentage in Defining Body Composition. Singapore Medical Journal, 53, 403-408.

[18] Courtney, J.J. and Janssen, I. (2007) Development of Age-Specific Adolescent Metabolic Syndrome Criteria That Are Linked to the Adult Treatment Panel III and International Diabetes Federation Criteria. Journal of the American College of Cardiology, 49, 891-898. https://doi.org/10.1016/j.jacc.2006.08.065

[19] Al-Hazzaa, H.M., Al-Sobayel, H.I., Qahwaji, D.M., Alsulaiman, N.A. and Musaiger, A.O. (2014) Prevalence of Overweight, Obesity, and Abdominal Obesity among Urban Saudi Adolescents: Gender and Regional Variations. Journal of Health Population Nutrition, 32, 634-645.

[20] Al-Nuaim, A.R., Bamgboye, E.A. and Al-Herbish, A. (1996) The Pattern of Growth and Obesity in Saudi Arabian Male School Children. International Journal of Obesity and Related Metabolic Disorders, 20, 1000-1005.

[21] Katzmarzyk, P.T. (2007) Epidemiology of Obesity. Canadian Medical Association 
Journal, 176, 18-29.

[22] Al-Hazzaa, H.M. (2002) Physical Activity, Fitness and Fatness among Saudi Children and Adolescents. Saudi Medical Journal, 23, 144-150.

[23] Janssen, I., Katzmarzyk, P.T., Boyce, W.F., Vereecken, C., Mulvihill, C., Roberts, C., et al. (2005) Health Behaviour in School-Aged Children Obesity Working Group. Comparison of Overweight and Obesity Prevalence in School-Aged Youth from 34 Countries and Their Relationships with Physical Activity and Dietary Patterns. Obesity Review, 6, 123-132. https://doi.org/10.1111/j.1467-789X.2005.00176.x

[24] Nicklas, T.A., Baranowski, T., Cullen, K.W. and Berenson, G. (2001) Eating Pattern, Dietary Quality and Obesity. The Journal of the American College of Nutrition, 20, 599-608. https://doi.org/10.1080/07315724.2001.10719064

[25] Abalkhail, B. and Shawky, S. (2002) Prevalence of Daily Breakfast Intake, Iron Deficiency Anaemia and Awareness of Being Anaemic among Saudi School Students. International Journal of Food Sciences and Nutrition, 53, 519-528. https://doi.org/10.1080/09637480220164370

[26] Al-Othaimeen, A., Osman, A.K. and Al Orf, S. (1999) Prevalence of Nutritional Anaemia among Primary School Girls in Riyadh City, Saudi Arabia. International Journal of Food Sciences and Nutrition, 50, 237-243. https://doi.org/10.1080/096374899101111

[27] Utter, J., Scragg, R., Schaaf, D. and Mhurchu, C.N. (2008) Relationships between Frequency of Family Meals, BMI and Nutritional Aspects of the Home Food Environment among New Zealand Adolescents. International Journal of Behavioral $\mathrm{Nu}$ trition and Physical Activity, 5, 50. https://doi.org/10.1186/1479-5868-5-50

[28] Minehira, K., Bettschart, V., Vidal, H., Vega, N., Di Vetta, V., Rey, V., Schneiter P., et al. (2003) Effect of Carbohydrate Overfeeding on Whole Body and Adipose Tissue Metabolism in Humans. Obesity Review, 11, 1096-1103. https://doi.org/10.1038/oby.2003.150

[29] Basciano, H., Fedserico, L. and Deli, K. (2005) Fructose, Insulin Resistance, and Metabolic Dyslipidemia. Nutrition Metabolism, 2, 5-14.

https://doi.org/10.1186/1743-7075-2-5

[30] Bleich, S.N., Wolfson, J.A., Vine, S. and Wang, Y.C. (2014) Diet-Beverage Consumption and Caloric Intake among US Adults, Overall and by Body Weight. American Journal of Public Health, 104, e72-e78. https://doi.org/10.2105/AJPH.2013.301556

[31] Heaney, R.P., Davies, K.M. and Barger-Lux, M.J. (2002) Calcium and Weight: Clinical Studies. The Journal of the American College of Nutrition, 21, 152S-155S. https://doi.org/10.1080/07315724.2002.10719213

[32] Pereira, M.A., Jacobs, D.R., Van Horn, L., Slattery, M.L., Kartashov, A.I. and Ludwig, D.F.S. (2002) Dairy Consumption, Obesity, and Insulin Resistance Syndrome in Young Adults: The CARDIA Study. Journal of the American Medical Association, 287, 2081-2089. https://doi.org/10.1001/jama.287.16.2081 
Submit or recommend next manuscript to SCIRP and we will provide best service for you:

Accepting pre-submission inquiries through Email, Facebook, LinkedIn, Twitter, etc. A wide selection of journals (inclusive of 9 subjects, more than 200 journals)

Providing 24-hour high-quality service

User-friendly online submission system

Fair and swift peer-review system

Efficient typesetting and proofreading procedure

Display of the result of downloads and visits, as well as the number of cited articles Maximum dissemination of your research work

Submit your manuscript at: http://papersubmission.scirp.org/

Or contact fns@scirp.org 